\title{
7
}

\section{Stabilizing PID Controllers for a Class of Time Delay Systems}

\author{
Karim Saadaoui, Sami Elmadssia and Mohamed Benrejeb \\ UR LARA-Automatique, Ecole Nationale d'Ingénieurs de Tunis, \\ University of Tunis, ElManar \\ Tunisia
}

\section{Introduction}

It is well known that delay exists in many practical systems such as industrial systems, biological systems, engineering systems, population dynamic models, etc... Time delay may appear as part of the internal dynamics or may result because of the actuators and sensors used. Communication delays between different parts of a system are also sources of this phenomenon. Existence of time delays may cause oscillations and even instability; moreover it complicates the analysis and control of these systems (Zhong, 2006; Normey-Rico \& Camacho, 2007). For all these reasons there is an extensive literature on stability analysis, stabilization and control of time delay processes, which continue to be active areas of research.

Recently, there has been a great interest in stabilizing high order systems by low fixed order controllers. The main motivation for using low order controllers comes from their simplicity and practicality, which explains the desire to reduce controller complexity and to determine as low order a controller as possible for a given high order or complex plant.

Computational methods for determining the set of all stabilizing controllers, of a given order and structure, for linear time invariant delay free systems are reported in the literature (Saadaoui \& Ozguler, 2005; Silva, 2005). In this line of research, the main objective is to compute the stabilizing regions in the parameter space of simple controllers since stabilization is a first and essential step in any design problem. Once the set of all controllers of a given order and structure is determined, further design criteria can be added. To this end, several approaches are employed, among which extensions of the Hermite-Biehler theorem and the D-decomposition method seem to be the most appropriate. Stabilization of special classes of time delay systems by fixed-order controllers was also addressed. Using a generalization of the Hermite-Biehler theorem the set of all stabilizing PI and PID controllers are determined for first order systems with dead time (Silva et al., 2001; Silva et al., 2002). Graphical methods were used to determine all stabilizing parameters of a PID controller applied to second order plants with dead time (Wang, 2007b).

In this chapter, we consider determining all stabilizing PID controllers for $n$-th order all poles systems with time delay. These systems can be used to model many physical examples. This includes the representation of a ship positioning an underwater vehicle 
through a long cable (Zhong, 2006). It can be used to represent the dynamic behavior of temperature control in a mix process (Normey-Rico \& Camacho, 2007). One of the main contributions of this work is the determination of admissible values of one of the controller's parameters. This is done using two approaches, the first approach is based on the use of an extension of the Hermite-Biehler theorem and the second approach is characterized by the application of a necessary condition which is a consequence of Kharitonov's lemma. The Ddecomposition method is then applied to find stabilizing regions in the parameter space of the remaining two parameters. By sweeping over the admissible ranges of the first parameter, the complete set of stabilizing PID controllers for this class of systems is obtained. It can be easily shown that the proposed method is applicable to first order controllers, being phase lead or phase lag controllers, by carrying out the appropriate modifications.

Stabilization being the most basic requirement in most controller design problems, determining the set of all stabilizing PID controllers for this class of systems is a first step in searching, among such controllers, those that satisfy further performance criteria, such as those imposed on the unit step response of the closed loop system. Once this first step is done, genetic algorithms are used to minimize several performance measures of the closed loop system. Genetic algorithms (GAs) (Chen et al., 2009; Chen \& Chang, 2006; Jan et al., 2008 ; Goldberg, 1989; Haupt \& Haupt, 2004; Melanie, 1998) are stochastic optimization methods. Unlike classical optimization methods, they are not gradient based which makes GAs suitable to minimize performance measures such as maximum percent overshoot, rise time, settling time and integral square error. Moreover, genetic algorithms explore the entire admissible space to search the optimal solution. This is another reason for using GAs after finding the complete set of stabilizing PID controllers which form the set of admissible solutions. In this work, the ranges of proportional, derivative and integral gains are not set arbitrary but they are set within the stabilizing regions determined, so that we are searching among the stabilizing values of the PID controller.

The chapter is organized as follows. In Section 2, a constant gain stabilizing algorithm is used to determine the admissible ranges of one of the controller's parameters. Next, the Ddecomposition method is introduced and a necessary condition is used to compute the admissible ranges of proportional and derivative gains. In Section 3, the stabilizing regions in the space of the controller's parameters are determined and the genetic algorithm method is used to minimize several performance measures of the step response of the closed loop system. In Section 4, illustrative examples are given. Finally, Section 6 contains some concluding remarks.

\section{Determining controller's stabilizing parameters}

In this work, we consider determining the stabilizing regions in the parameter space of a PID controller,

$$
C(s)=\frac{k_{d} s^{2}+k_{p} s+k_{i}}{s}
$$

Applied to an $\mathrm{n}$-th order all poles system with delay, 


$$
G(s)=\frac{e^{-L s}}{Q(s)}=\frac{e^{-L s}}{q_{n} s^{n}+\ldots+q_{0}}
$$

Where $L>0$ represents the time delay. In this section, the admissible ranges of the parameters $\left(k_{p}, k_{d}\right)$ are found, where $k_{p}$ denotes the proportional gain and $k_{d}$ the derivative gain. The exact range of stabilizing $\left(k_{p}, k_{d}\right)$ values is difficult to determine analytically. In fact, the problem of determining the exact range of stabilizing $k_{p}$ values analytically is solved for the case of a first order plant with dead time (Silva et al., 2002). Therefore, instead of determining the exact range of $k_{p}$ ( or $k_{d}$ ) a necessary condition will be used to get an estimate of the stabilizing range.

Let us first fix the notation used in this work. Let $R$ denote the set of real numbers and $C$ denote the set of complex numbers and let $R_{-}, R_{0}$ and $R_{+}$denote the points in the open left-half, $j w$-axis and the open right-half of the complex plane, respectively. The derivative of a polynomial or a quasi-polynomial $\Psi$ is denoted by $\Psi^{\prime}$. The set $H$ of stable polynomials or quasi-polynomials are,

$$
H=\left\{\Psi(s) \in R[s]: \Psi(s)=0 \Rightarrow s \in C_{-}\right\}
$$

Given a set of polynomials $\Psi_{1}, \cdots, \Psi_{l} \in R[s]$ not all zero and $l>1$ their greatest common divisor is unique and it is denoted by $\operatorname{gcd}\left\{\Psi_{1}, \cdots, \Psi_{l}\right\}$; If $\operatorname{gcd}\left\{\Psi_{1}, \cdots, \Psi_{l}\right\}=1$ then we say $\left\{\Psi_{1}, \cdots, \Psi_{l}\right\}$ is coprime. The signature $\sigma(\Psi)$ of a polynomial $\Psi \in R[s]$ is the difference between the number of its $C_{-}$roots and $C_{+}$roots. Given $\Psi \in R[s]$ the even-odd components $(a, b)$ of $\Psi(s)$ are the unique polynomials $a, b \in R\left[s^{2}\right]$ such that

$$
\Psi(s)=a\left(s^{2}\right)+s b\left(s^{2}\right)
$$

It is possible to state a necessary and sufficient condition for the Hurwitz stability of $\Psi(s)$ in terms of its even-odd components $(a, b)$. Stability is characterized by the interlacing property of the real, negative, and distinct roots of the even and odd parts. This result is known as the Hermite-Biehler theorem. Below is a generalization of the Hermite-Biehler theorem applicable to not necessarily Hurwitz stable polynomials. Let us define the signum function $S: R \rightarrow\{-1,0,1\}$ by

$$
S u=\left\{\begin{array}{cc}
-1 & \text { if } u<0 \\
0 & \text { if } u=0 \\
1 & \text { if } u>0
\end{array}\right.
$$

Lemma 1. (Saadaoui \& Ozguler, 2003) Let a nonzero polynomial $\Psi \in R[s]$ has the even-odd components $(a, b)$ Suppose $b \neq 0$ and $(a, b)$ is coprime. Then, $S(\Psi)=r$ if and only if at the real negative roots of odd multiplicities $v_{1}>v_{2}>\cdots>v_{l}$ of $b$ the following holds:

$$
r=\left\{\begin{array}{cl}
S b\left(0_{-}\right)\left[S a(0)-2 S a\left(v_{1}\right)+2 S a\left(v_{1}\right)+\cdots+(-1)^{l} 2 S a\left(v_{l}\right)\right] & \text { if } \operatorname{deg} \Psi \text { odd } \\
S b\left(0_{-}\right)\left[S a(0)-2 S a\left(v_{1}\right)+2 S a\left(v_{1}\right)+\cdots+(-1)^{l+1} S a(-\infty)\right] & \text { if } \operatorname{deg} \Psi \text { even }
\end{array}\right.
$$


where $S b\left(0_{-}\right):=(-1)^{m 0} b^{(m 0)}(0), m 0$ is the multiplicity of $u=0$ as a root of $b(u)$, and $b^{(m 0)}(0)$ denotes the value at $u=0$ of the $m 0$-th derivative of $b(u)$.

The following result, determines the number of real negative roots of a real polynomial.

Lemma 2. (Saadaoui \& Ozguler, 2005) A nonzero polynomial $\Psi \in R[s]$, such that $\Psi(0) \neq 0$, has $r$ real negative roots without counting the multiplicities if and only if the signature of the polynomial $\Psi\left(s^{2}\right)+\Psi^{\prime}\left(s^{2}\right)$ is $2 r$. All roots of $\psi$ are real, negative, and distinct if and only if $\Psi\left(s^{2}\right)+\Psi^{\prime}\left(s^{2}\right) \in H$.

We now describe a slight extension of the constant stabilizing gain algorithm of (Saadaoui \& Ozguler,2003). Given a plant

$$
G_{0}(s)=\frac{p_{0}(s)}{q_{0}(s)}
$$

where $p_{0}, q_{0} \in R[s]$ are coprime with $m=\operatorname{deg} p_{0}$ less than or equal to $n=\operatorname{deg} q_{0}$, the set

$$
\Phi_{r}\left(p_{0}, q_{0}\right)=\left\{\alpha \in R: \sigma[\varphi(s, \alpha)]=\sigma\left[q_{0}(s)+\alpha p_{0}(s)\right]=r\right\}
$$

is the set of all real $\alpha$ such that $\phi(s, \alpha)$ has signature equal to $r$. Let $(h, g)$ and $(f, e)$ be the even-odd components of $q_{0}(s)$ and $p_{0}(s)$, respectively, so that

$$
\begin{aligned}
& q_{0}(s)=h\left(s^{2}\right)+s g\left(s^{2}\right), \\
& p_{0}(s)=f\left(s^{2}\right)+s e\left(s^{2}\right) .
\end{aligned}
$$

Let $(H, G)$ be the even-odd components of $q_{0}(s) p_{0}(-s)$. Also let $F\left(s^{2}\right)=p_{0}(s) p_{0}(-s)$. By a simple computation, it follows that $\left(s^{2}\right.$ is replaced by $\left.u\right)$ :

$$
\begin{gathered}
H(u)=h(u) f(u)-u g(u) e(u) \\
G(u)=g(u) f(u)-h(u) e(u) \\
F(u)=f^{2}(u)-u e^{2}(u)
\end{gathered}
$$

With this setting, we have

$$
\left[q_{0}(s)+\alpha p_{0}(s)\right] p_{0}(-s)=\left[H\left(s^{2}\right)+\alpha F\left(s^{2}\right)\right]+s G\left(s^{2}\right)
$$

If $G \neq 0$ and if they exist, let the real negative roots with odd multiplicities of $G(u)$ be $\left\{v_{1}, v_{2}, \cdots v_{l}\right\}$ with the ordering $v_{1}>v_{2}>\cdots>v_{l}$, with $v_{0}:=0$ and $v_{l+1}:=-\infty$ for notational convenience. 
The following algorithm determines whether $\Phi_{r}\left(p_{0}, q_{0}\right)$ is empty or not and outputs its elements when it is not empty (Saadaoui \& Ozguler, 2005) :

\section{Algorithm 1.}

1. Consider all the sequences of signums

$$
I=\left\{\begin{array}{cc}
\left\{i_{0}, i_{1}, \cdots, i_{l}\right\} & \text { for odd } r-m \\
\left\{i_{0}, i_{1}, \cdots, i_{l+1}\right\} & \text { for even } r-m
\end{array}\right.
$$

where

$$
i_{j} \in\{-1,1\} \text { for } j=0,1, \cdots, l+1 .
$$

2. Choose all the sequences that satisfy

$$
r-\sigma\left(p_{0}\right)=\left\{\begin{array}{cc}
i_{0}-2 i_{1}+2 i_{2} \cdots+2(-1)^{l} i_{l} & \text { for odd } r-m \\
i_{0}-2 i_{1}+2 i_{2} \cdots+(-1)^{l+1} i_{l+1} & \text { for even } r-m
\end{array} .\right.
$$

3. For each sequence of signums $I\left\{i_{j}\right\}$ that satisfy step 2, let

$$
\alpha_{\max }=\max \left\{-\frac{H}{V}\left(v_{j}\right)\right\} \quad \forall v_{j} \text { forwhich } F\left(v_{j}\right) \neq 0 \text { and } i_{j} S F\left(v_{j}\right)=1
$$

and

$$
\alpha_{\min }=\min \left\{-\frac{H}{V}\left(v_{j}\right)\right\} \quad \forall v_{j} \text { forwhich } F\left(v_{j}\right) \neq 0 \text { and } i_{j} S F\left(v_{j}\right)=-1 .
$$

The set $\Phi_{r}\left(p_{0}, q_{0}\right)$ is non-empty if and only if for at least one signum sequence I satisfying step 2, $\alpha_{\max }<\alpha_{\min }$ holds.

4. $\Phi_{r}\left(p_{0}, q_{0}\right)$ is equal to the union of intervals $\left(\alpha_{\max }, \alpha_{\min }\right)$ for each sequence of signums I that satisfy step 3 .

The algorithm above is easily specialized to determine all stabilizing proportional controllers $C(s)=\alpha$ for the plant $G_{0}(s)$. This is achieved by replacing $r$ in step 2 of the algorithm by $n$, the degree of $\phi(s, \alpha)$.

Remark 1. By Step 2 of Algorithm 1, a necessary condition for the existence of a $\alpha \in \Phi_{r}\left(p_{0}, q_{0}\right)$ is that the odd part of $\left[q_{0}(s)+\alpha p_{0}(s)\right] p_{0}(s)$ has at least $\bar{r}=\left\lfloor\frac{\left|r-\sigma\left(p_{0}\right)\right|-1}{2}\right\rfloor$ real negative roots with odd multiplicities. When solving a constant stabilization problem, this lower bound is $\bar{r}=\left\lfloor\frac{r-\sigma\left(p_{0}\right)-1}{2}\right\rfloor$. 


\subsection{Determining the admissible ranges of $k_{p}$ using padé approximation}

In this part, our aim is to find all admissible values of $k_{p}$. Replacing the time delay by a Padé approximation $e^{-L s} \approx \frac{p(-s)}{p(s)}$ where $p(s) \in H$, we get the following closed-loop characteristic polynomial

$$
\begin{aligned}
\phi_{0}(s) & =s q(s) p(s)+\left(k_{d} s^{2}+k_{p} s+k_{i}\right) p(-s) \\
& =q_{0}(s)+\left(k_{d} s^{2}+k_{p} s+k_{i}\right) p_{0}(s)
\end{aligned}
$$

Where

$$
\begin{gathered}
q_{0}(s)=s q(s) p(s), \\
p_{0}(s)=p(-s) .
\end{gathered}
$$

Multiplying $\phi_{0}(s)$ by $p_{0}(-s)$, we obtain

$$
\begin{aligned}
\psi_{0}(s) & =\phi_{0}(s) p_{0}(-s) \\
& =\left(H(.)+s^{2} k_{d} F(.)+k_{i} F(.)\right)+s\left(G(.)+k_{p} F(.)\right)
\end{aligned}
$$

where $H, G$ and $F$ are given by (4). Note that $p_{0}(-s)=p(s) \in H$, therefore by Remark 1 the odd part $G(u)+k_{p} F(u)$ of $\psi_{0}(s)$ must have all its roots real, negative, and distinct. At this step, two parameters $k_{d}$ and $k_{i}$ are eliminated and an auxiliary problem with only one parameter will be solved. Let

$$
\varphi_{1}(s)=\left(G\left(s^{2}\right)+s G^{\prime}\left(s^{2}\right)\right)+k_{p}\left(F\left(s^{2}\right)+F^{\prime}\left(s^{2}\right)\right)
$$

using Lemma 2, finding values of $k_{p}$ such that $G(u)+k_{p} F(u)$ has all its roots real, negative and distinct is equivalent to stabilizing the new constructed polynomial $\varphi_{1}(s)$. This new constructed problem can be solved using Algorithm 1 as only one parameter appears.

\subsection{An alternative method for computing the admissible ranges of $k_{p}$}

In this part an alternative method is used to get the admissible values of $k_{p}$ without the need of using Padé approximation. Moreover this method allows the calculation of the admissible ranges of the parameters $\left(k_{p}, k_{d}\right)$, where $k_{p}$ denotes the proportional gain and $k_{d}$ the derivative gain. The following result will be used in determining the admissible values of $k_{p}$ (alternatively $k_{d}$ ).

Lemma 3: (Kharitonov et al., 2005) Consider the quasi-polynomial,

$$
\Psi(s)=\sum_{i=0}^{n} \sum_{l=1}^{r} h_{i l} s^{n-i} e^{\tau_{l} s}
$$


such that $\tau_{1}<\tau_{2}<\cdots<\tau_{r}$, with main term $h_{0 r} \neq 0$, and $\tau_{1}+\tau_{r}>0$. If $\Psi(s)$ is stable, then $\Psi^{\prime}(s)$ is also a stable quasi-polynomial.

The characteristic function of the closed loop system formed by the PID controller and the time delay system (2) is given by,

$$
\Psi_{1}(s)=s Q(s)+\left(k_{d} s^{2}+k_{p} s+k_{i}\right) e^{-L s}
$$

Since the term $e^{-L s}$ has no finite roots, the quasi-polynomial $\Psi_{1}(s)$ and $\Psi(s)=\Psi_{1}(s) e^{L s}$ have the same roots, therefore stability of $\Psi(s)$ is equivalent to stability of $\Psi_{1}(s)$, see (Kharitonov et al., 2005). In the sequel, the quasi-polynomial,

$$
\Psi(s)=s Q(s) e^{L s}+\left(k_{d} s^{2}+k_{p} s+k_{i}\right)
$$

will be used to study stability of the closed loop system. Now, using Lemma 3, if $\Psi(s)$ is stable then $\Psi^{\prime}(s)$ is also a stable quasi-polynomial, where $\Psi^{\prime}(s)$ is given by,

$$
\Psi^{\prime}(s)=\left((L s+1) Q(s)+s Q^{\prime}(s)\right) e^{L s}+2 k_{d} s+k_{p}
$$

Note that only two parameters $\left(k_{p}, k_{d}\right)$ appear in the expression of $\Psi^{\prime}(s)$. By Lemma 3, stabilizing $\Psi^{\prime}(s)$ is equivalent to calculating the admissible $\left(k_{p}, k_{d}\right)$ values for the original problem.

The D-decomposition method (Hohenbicher \& Ackermann, 2003; Gryazina \& Polyak, 2006) is used to determine the stabilizing regions in the $\left(k_{p}, k_{d}\right)$ plane. The D-decomposition method is based on the fact that roots of the quasi-polynomial (9) change continuously when the coefficients are changed continuously. Hence, a stable quasi-polynomial can become unstable if and only if at least one of its roots crosses the imaginary axis. Using this fact, the $\left(k_{p}, k_{d}\right)$ plane can be partitioned into regions having the same number of roots of (9) in the left half plane. Stability can be checked by choosing a point inside a region and applying classical methods such as the Nyquist criterion.

Evaluating the characteristic function at the imaginary axis is equivalent to replacing $s$ by $j \omega, \omega \geq 0$, in (9) we get,

$$
\begin{aligned}
& \Psi^{\prime}(j w)=\left[R(w)-L w I(w)-w I^{\prime}(w)\right]-\left[w L R(w)+w R^{\prime}(w)\right] \sin (L w)+k_{p} \\
& {\left[\left(R(w)-L w I(w)-w I^{\prime}(w)\right) \sin (L w)+\left(w L R(w)+w R^{\prime}(w) \cos (L w)+2 w k_{d}\right]\right.}
\end{aligned}
$$

where,

$$
Q(j \omega)=R(\omega)+j I(\omega)
$$

and,

$$
Q^{\prime}(j \omega)=R^{\prime}(\omega)+j I^{\prime}(\omega)
$$


Applying the D-decomposition method implies equating the real and imaginary parts of (10) to zero. Two cases must be considered:

- Case 1: $\omega=0$ which leads to the following equation,

$$
k_{p}=-Q(0)
$$

- Case 2: $\omega>0$ in this case we get,

$$
\begin{gathered}
k_{p}=-\left[R(w)-L w I(w)-w I^{\prime}(w)\right] \cos (L w) \\
+\left[w L R(w)+w R^{\prime}(w)\right] \sin (L w) \\
k_{d}=\left[\left(R(\omega)-L \omega I(\omega)-\omega I^{\prime}(\omega)\right) \sin (L \omega)\right. \\
\left.+\left(\omega L R(\omega)+\omega R^{\prime}(\omega)\right) \cos (L \omega)\right](2 \omega)^{-1}
\end{gathered}
$$

By sweeping over values of $\omega>0$, the $\left(k_{p}, k_{d}\right)$ plane can be partitioned into root invariant regions. Using (13), (14) and (15) stabilizing regions in the $\left(k_{p}, k_{d}\right)$ plane can be determined.

\section{PID controller design}

In this section, first the set of all stabilizing PID controllers are determined, this forms the set of admissible solutions for optimization. Next, GAs are used to optimize these PID parameters.

\subsection{Stabilizing regions}

The admissible $\left(k_{p}, k_{d}\right)$ values are calculated in section 2 , now, we go back to the original problem by considering (7). Our method consists of fixing one parameter $k_{p}$ or $k_{d}$ and determining the stabilizing regions in the plane of the remaining two parameters. By sweeping over the admissible values of the first parameter $\left(k_{p}\right.$ or $\left.k_{d}\right)$ the complete set of the stabilizing regions is found. Once again the D-decomposition method is used.

For $\omega=0$, we get the following equation,

$$
k_{i}=0
$$

For $\omega>0$, two cases will be investigated. First, let us fix $k_{p}$ and find the stabilizing regions in the $\left(k_{d}, k_{i}\right)$ plane. Using (7), replacing $s$ by $j \omega$ and equating the real and imaginary parts to zero we get,

$$
\left[\begin{array}{cc}
\cos (L \omega) & -\omega^{2} \cos (L \omega) \\
\sin (L \omega) & -\omega^{2} \sin (L \omega)
\end{array}\right]\left[\begin{array}{l}
k_{i} \\
k_{d}
\end{array}\right]=\left[\begin{array}{c}
k_{p} \omega \sin (L \omega)+\omega I(\omega) \\
-k_{p} \omega \cos (L \omega)+\omega R(\omega)
\end{array}\right]
$$

Note that the matrix at the left-hand of (17) is singular. The singular frequencies (Hohenbicher \& Ackermann, 2003) are determined as the solutions of equation (18), 


$$
k_{p}+I(\omega) \sin (L \omega)-R(\omega) \cos (L \omega)=0
$$

and are denoted by $\omega_{i}, i=1,2, \cdots$. For each singular frequency $\omega_{i}$, an equation of a straight line in the $\left(k_{d}, k_{i}\right)$ plane is defined by,

$$
k_{i}=\omega_{i}^{2} k_{d}+\omega_{i} R\left(\omega_{i}\right) \sin \left(L \omega_{i}\right)+\omega_{i} I\left(\omega_{i}\right) \cos \left(L \omega_{i}\right)
$$

which partition the plane into root-invariant regions among which the stabilizing regions, if any, have to be determined. Alternatively, one may fix $k_{d}$ first and repeat the above procedure. In this case we have,

$$
\left[\begin{array}{cc}
\cos (L \omega) & -\omega \sin (L \omega) \\
\sin (L \omega) & \omega \cos (L \omega)
\end{array}\right]\left[\begin{array}{l}
k_{i} \\
k_{p}
\end{array}\right]=\left[\begin{array}{l}
k_{d} \omega^{2} \cos (L \omega)+\omega I(\omega) \\
k_{d} \omega^{2} \sin (L \omega)-\omega R(\omega)
\end{array}\right]
$$

solving this system we get,

$$
\begin{aligned}
& k_{i}(\omega)=\omega^{2} k_{d}+\omega I(\omega) \cos (L \omega)-\omega R(\omega) \sin (L \omega) \\
& k_{p}(\omega)=-I(\omega) \sin (L \omega)-R(\omega) \cos (L \omega)
\end{aligned}
$$

For $\omega>0$, the above two equations partition the $\left(k_{p}, k_{i}\right)$ plane into root-invariant regions. Hence stabilizing regions, if any, can be found. It is interesting to note here that depending on which parameter we fix first, either $k_{p}$ or $k_{d}$, the obtained stabilizing region is different. This can be explained by the fact that in the first case the matrix is always singular and in the second case it is always non-singular.

\subsection{PID controller design using GAs}

Determining the total set of stabilizing PID controllers is a first step in the design process. Once this set of stabilizing PID controllers is found, it is natural to search within this set, controllers that meet extra performance specifications. Four performance measures will be considered:

- Maximum percent overshoot (OS).

- $\quad$ Settling time (ST).

- $\quad$ Rise time (RT).

- Integral square error (ISE) ISE $=\int_{0}^{t_{f}}|e(t)|^{2} d t$, where $\mathrm{e}(\mathrm{t})$ is the error at time $\mathrm{t}$.

Optimization is done using genetic algorithms. The first step in this design procedure, which consists of determining the total set of stabilizing PID controllers, is very important. It enhances the application of the genetic algorithm by fixing the search space, unlike other works on optimizing PID controllers using GAs (Chen et al., 2009; Chen \& Chang, 2006; Jan et al., 2008) where the ranges of $\left(k_{p}, k_{i}, k_{d}\right)$ are set arbitrary. Moreover, it improves the optimization time and increases the chances of obtaining the global optimum. Optimization 
of the PID controllers is done by minimizing each of the four cost functions: maximum percent overshoot, settling time, rise time and integral square error. GAs tries to find the global optimum by evaluating many points of the solution space in each generation. There are mainly three operations: reproduction, crossover and mutation, used to form new generations until a termination condition is reached (Goldberg, 1989; Haupt \& Haupt, 2004; Melanie, 1998). In what follows, we briefly describe the parameters and tools used in the implementation of the genetic algorithm:

1. Since stability is an essential property for any control system, the search space consists of the stabilizing values of the PID controller which are determined in section 3.1. Population size is chosen to be 150 individuals per population. In the beginning the initial population is randomly chosen within the stabilizing values of the PID controller. These individuals are candidate solutions to the problem. The number of generations is chosen as 100 .

2. Evaluation of a generation is done by calculating the cost function for each individual. Since minimization problems are considered, the fitness function will be the inverse of the cost function.

3. Genetic operators: reproduction, crossover and mutation are used to form new generations. Individuals are chosen for reproduction according to their fitness value. Fittest ones have larger probability of selection. The Roulette Wheel selection method is used.

4. In order to improve next generations, crossover which is a process of combining parts of the parent individuals to produce new offsprings is done. Crossover value used is 0.8 .

To avoid local minimum and explore new parts of the search space, mutation process is applied. It consists of randomly modifying individuals in the generation.

1. Mutation probability is always set to a small value so that the search algorithm is not turned to a random search algorithm. The value chosen in our case is 0.01 .

2. Steps $2-5$ are repeated until a termination condition is reached. The maximum generation termination condition is adopted.

\section{Examples}

In this section three illustrative examples are given.

\section{Example 1:}

Consider stabilizing the second order integrating system,

$$
G(s)=\frac{11.32}{s^{2}+11.32 s} e^{-s}
$$

by a PID controller. This transfer function represents a system composed of master-slave parts. The slave part is a model of a mobile robot which can move in one direction (Seuret et al., 2006). This robot is controlled through a communication network which introduces delays in the control loop. See (Seuret et al., 2006) for more details about this system. First, 
the admissible values of $\left(k_{p}, k_{d}\right)$ are calculated, see Fig. 1 . The first approach proposed consists of fixing $k_{p}$ and using (16), (18) and (19) to determine stabilizing regions in the plane of the remaining two parameters.

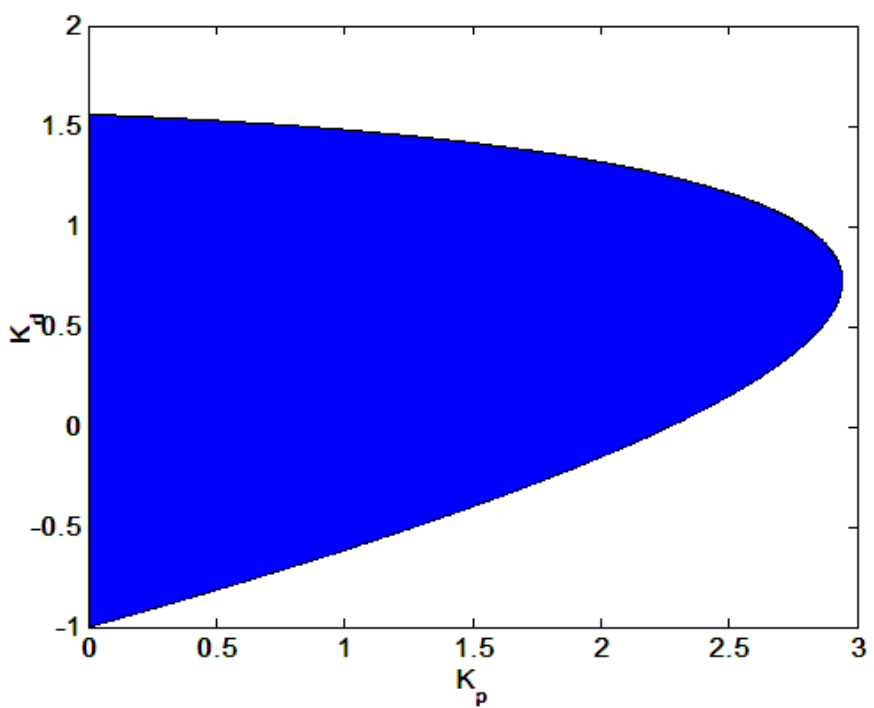

Fig. 1. Admissible values of $\left(k_{p}, k_{d}\right)$.

By sweeping over the admissible values of $k_{p}$, the complete stabilizing regions of $\left(k_{p}, k_{i}, k_{d}\right)$ values are found as shown in Fig.2.

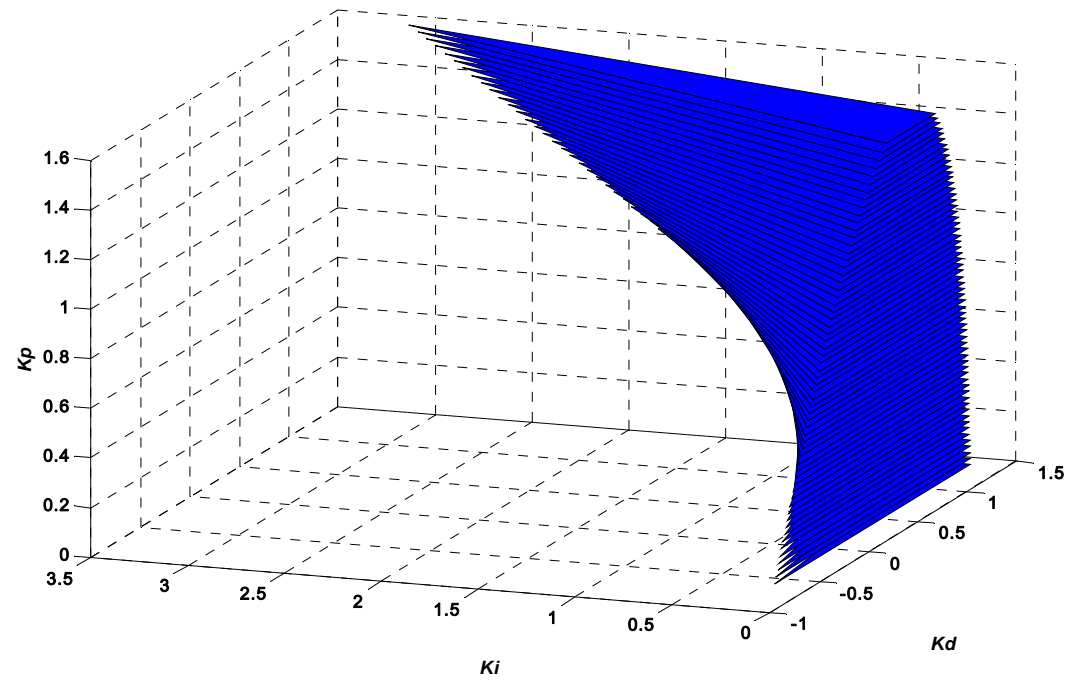

Fig. 2. Complete set of stabilizing PID controllers for example1. 
Alternatively, we can fix $k_{d}$ and use (16), (21) and (22) to find the stabilizing regions of $\left(k_{p}, k_{i}, k_{d}\right)$ values as shown in Fig.3.

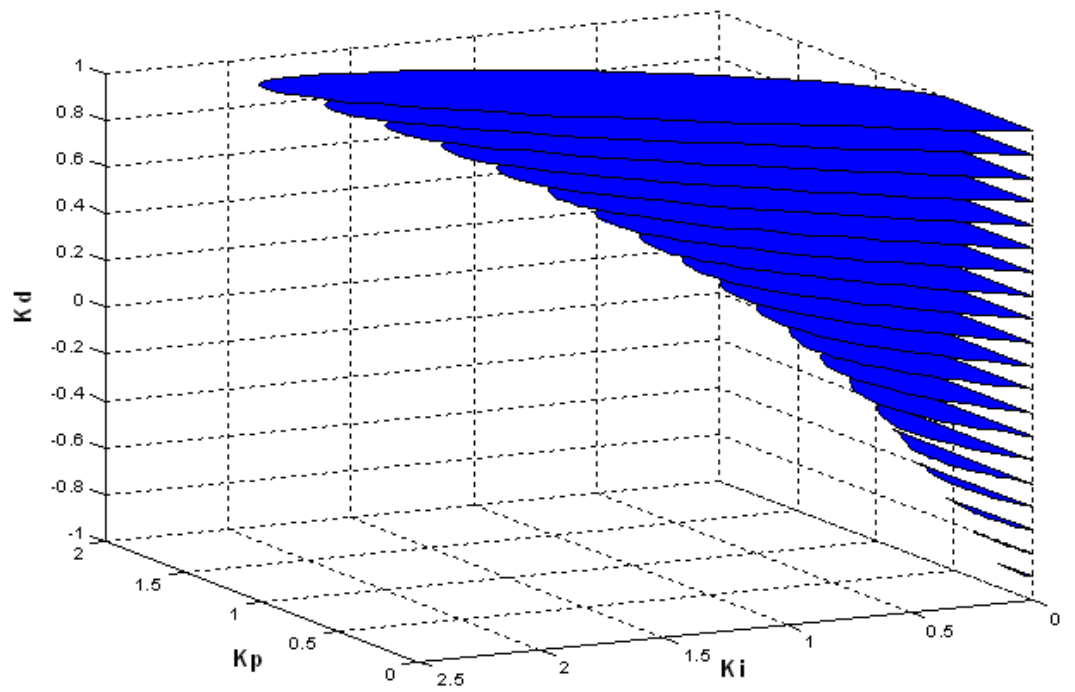

Fig. 3. Complete set of stabilizing PID controllers for example1.

Fixing $k_{d}=0$ is equivalent to using a PI controller. For $k_{d}=0$, we obtain the stabilizing region of $\left(k_{p}, k_{i}\right)$ values as given in Fig. 4 .

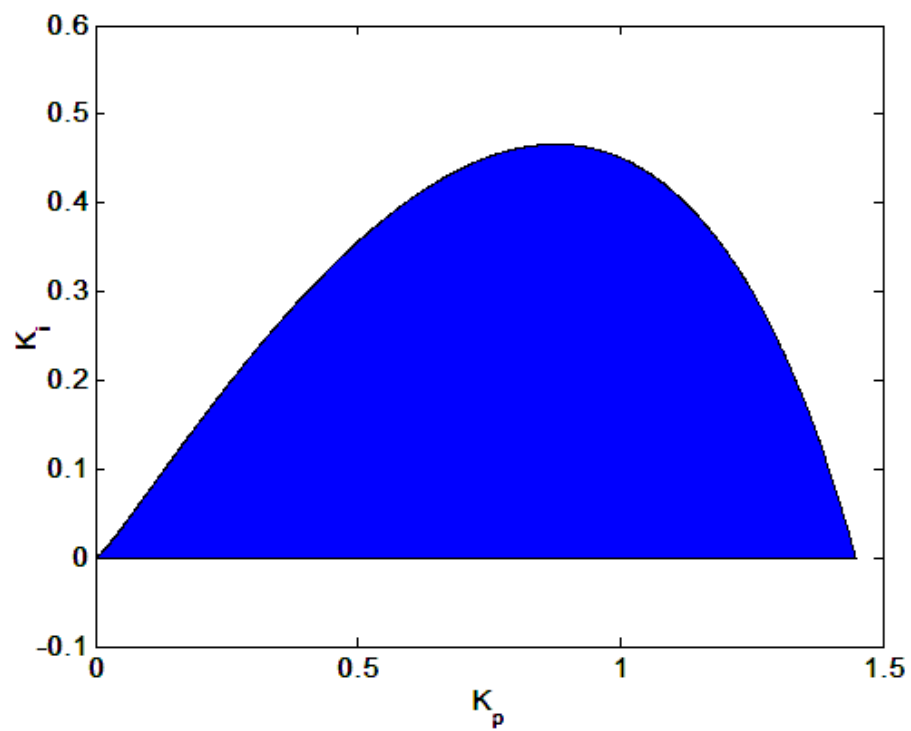

Fig. 4. Complete set of stabilizing PI controllers for example1. 
Using this stabilizing region of $\left(k_{p}, k_{i}\right)$ values, we apply the genetic algorithm described in section 3.2, to minimize each of the performance indices: maximum percent overshoot (Opt1), settling time (Opt2), rise time (Opt3) and integral square error (opt4). Although a simple controller, PI controller, is used for an integrating system with delay, the results obtained are satisfactory as shown by the step response of the closed loop system in Fig. 5.

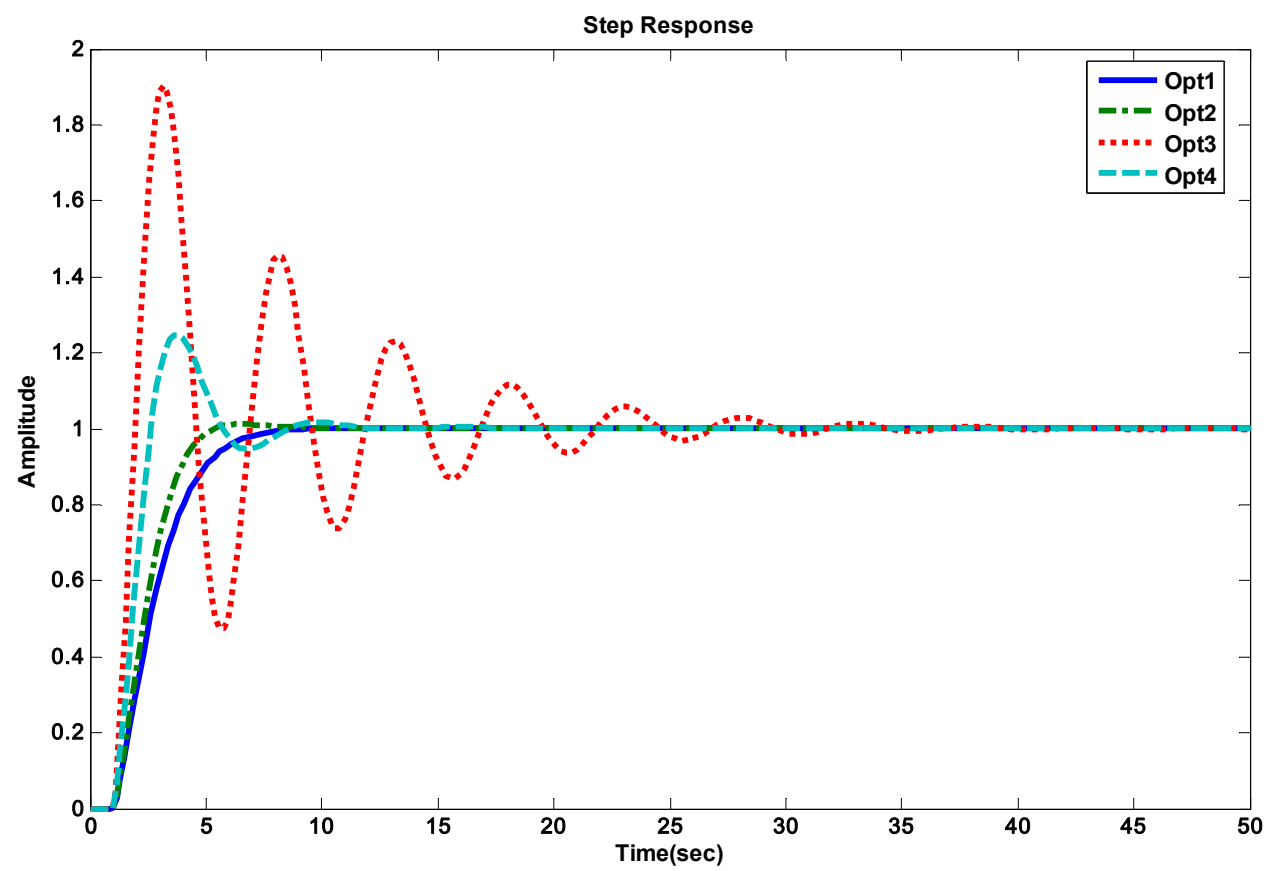

Fig. 5. Step responses of the closed loop system.

\section{Example 2:}

Consider stabilizing the first order system given in (Silva et al., 2005),

$$
G(s)=\frac{1}{3 s+1} e^{-2.8 s}
$$

by a PID controller. For comparison reasons, we apply three classical methods. These methods are: Ziegler-Nichols method $(\mathrm{ZN})$, Chien Hornes \& Reswich method (CHR) and finally Cohen-Coon method (CC). After obtaining the stabilizing regions of $\left(k_{p}, k_{i}, k_{d}\right)$ values, we apply the genetic algorithm to minimize the four performance indices described in section 3.2. Step responses of the closed loop system obtained with classical methods are given in Fig 6. 


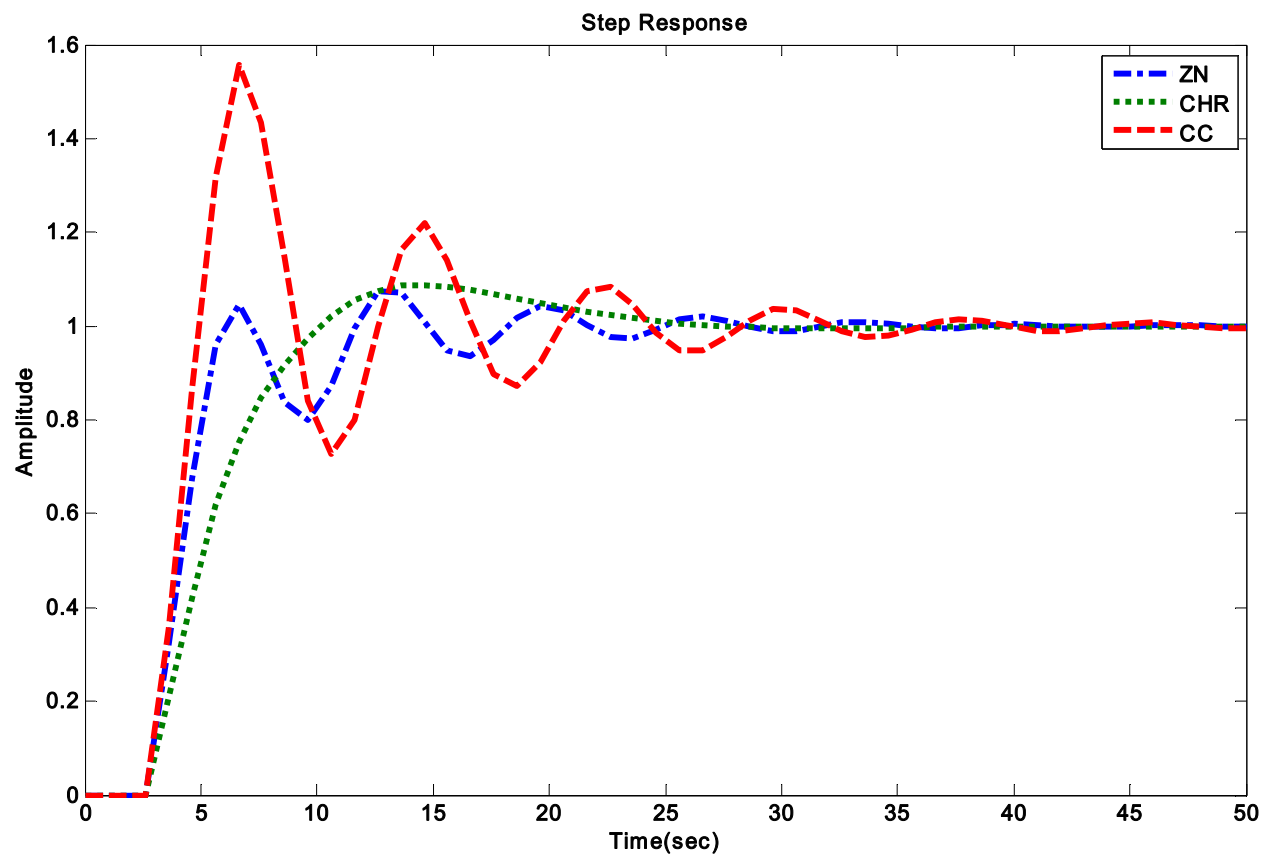

Fig. 6. Step responses of the closed loop system for example 2.

Determining the set of stabilizing controllers and using GAs to optimize these stabilizing values, gives the step responses shown in Fig 7.

Table 1 summarizes the different results obtained using our method and classical methods. For each performance criteria we can see that our method gives better results. However it should be stressed here that minimizing one performance criteria can deteriorate other performance indices, for example we minimize overshoot at the expense of a larger settling time as shown in the results below. Optimizing the integral square error (opt4) gives better results as an overall performance. Another advantage of our method is to answer the question: what is the best performance we can get with such a controller? This is possible as optimization is done over all the admissible stabilizing values of the controller. 


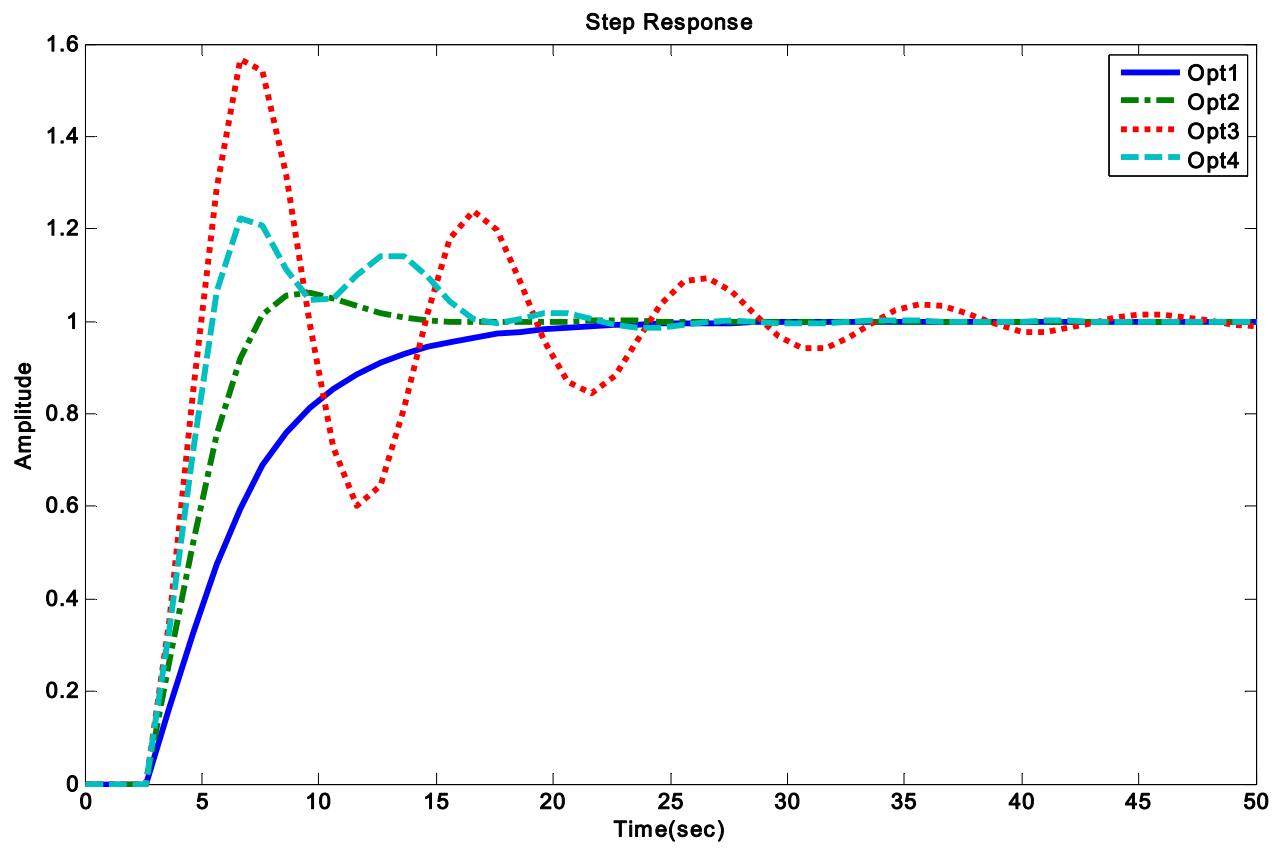

Fig. 7. Step responses of the closed loop system for example 2. 


\begin{tabular}{|l|c|c|c|c|c|}
\hline Method & $\left(\mathrm{K}_{\mathrm{p}}, \mathrm{K}_{\mathrm{i}}, \mathrm{K}_{\mathrm{d}}\right)$ & OS $(\%)$ & ST $(\mathrm{s})$ & RT (s) & ISE \\
\hline ZN & $(1.28,0.22,1.8)$ & 7.9 & 24.9 & 5.9 & 3.17 \\
\hline CHR & $(0.64,0.21,0.9)$ & 9.1 & 24 & 8 & 3.75 \\
\hline CC & $(1.69,0.39,1.49)$ & 51 & 32.9 & 5.8 & 3.33 \\
\hline Opt 1 & $(0.52,0.14,0.28)$ & 0 & 19 & 9 & 4.77 \\
\hline Opt 2 & $(0.84,0.22,0.39)$ & 4.9 & 13 & 7 & 3.73 \\
\hline Opt 3 & $(1.69,0.35,0.55)$ & 58 & 48 & 6 & 4.32 \\
\hline Opt 4 & $(1.22,0.35,1.67)$ & 21.7 & 21.9 & 5.9 & 2.95 \\
\hline
\end{tabular}

Table 1. Results obtained by different methods.

\section{Example 3:}

Consider stabilizing the second order plant given in (Wang, 2007a) by

$$
G(s)=\frac{k}{\left(1+T_{1} s\right)\left(1+T_{2} s\right)} e^{-L s},
$$

by a PID controller.

Let $k=1$ and $L=0.2$ applying the procedure developed in this work, we get (the four cases given in (Wang, 2007a) will be investigated):

- Case 1: For $T_{1}=0.2$ and $T_{2}=0.5$ the admissible range of $k_{p}$ values is obtained as $-1<k_{p}<5.56$ with a Padé approximation of order 3.

- Case 2: For $T_{1}=-1$ and $T_{2}=-0.5$ the admissible range of $k_{p}$ values is obtained as $-1<k_{p}<0.58$ with a Padé approximation of order 2 .

- Case 3: For $T_{1}=1$ and $T_{2}=-0.5$ the admissible range of $k_{p}$ values is obtained as $-5.21<k_{p}<-1$ with a Padé approximation of order 3 .

- Case 4: For $T_{1}=-1$ and $T_{2}=0.5$ the admissible range of $k_{p}$ values is obtained as $-9.79<k_{p}<-1$ with a Padé approximation of order 3 .

Although a necessary condition is used to determine the admissible ranges of $k_{p}$, in the four cases above the intervals obtained are the same as the ones given in (Wang, 2007a).

\section{Conclusion}

In this work, a new method is given for calculating all stabilizing values of a PID controller which is applied to a special class of time delay systems. The proposed approach is based on determining first the admissible ranges of one of the controller's parameters. This step is solved using two different approaches. Next, the D-decomposition method is applied to obtain the stabilizing regions in the controller's parameter space. The genetic algorithm optimization method is then applied to find among those stabilizing controllers those that satisfy further performance specifications. Four time domain measures, which are maximum 
percent overshoot, settling time, rise time and integral square error, were minimized. Application of the proposed method to uncertain time delay system is under investigation.

\section{References}

Chen, C. K.; Koo, H. H.; Yan, J. J. \& Liao, T. L. (2009). GA-based PID active queue management control design for a class of TCP communication networks, Expert Systems with Applications, Vol. 36, pp. 1903-1913.

Chen, C. K. \& Chang, S. H. (2006). Genetic algorithms based optimization design of a PID controller for an active magnetic bearing. International Journal of Computer Science and Network, Vol. 6, pp. 95-99.

Datta, A.; Ho, M. T. \& Bhattacharyya, S. P. (2000). Structure and synthesis of PID controller, Springer-Verlag, ISBN 1-85233-614-5, London, Great Britain.

Goldberg, D. E. (1989). Genetic algorithms in search, optimization and machine learnin, AddisonWesley, ISBN 0201157675.

Gryaznia, E. N. \& Polyak, B. T. (2006). Stability regions in the parameter space: Ddecomposition revisited. Automatica, Vol. 42, pp. 13-26.

Haupt, R. L. \& Haupt, S. E. (2004) Practical genetic algorithms, John Wiley \& Sons, ISBN 0471188735.

Hohenbicher, N. \& Ackermann, J. (2003). Computing stable regions in parameter spaces for a class of quasi-polynomials, Proc. 4th IFAC Workshop on Time Delay Systems TDS'03, Recquencourt, France.

Jan, R. M.; Tseng, C. S. \& Lin, R. J. (2008). Robust PID control design for permanent magnet synchronous motor: A genetic approach. Electric Power Systems Research, Vol. 78, pp. 1161-1168.

Melanie, M. (1998) An introduction to genetic algorithms, MIT Press, ISBN 0262631857.

Normey-Rico, J. E. \& Camacho, E. F. (2007). Control of dead time process. Springer, London.

Kharitonov, V. L.; Niculescu, S.; Moreno, J. \& Michiels, W. (2005). Static output stabilization: Necessary conditions for multiple delay controllers. IEEE Transactions on Automatic Control, Vol. 50, pp. 82-86.

Saadaoui, K. \& Ozguler, A. B. (2003). On the set of all stabilizing first order controllers, Proc. American Control Conference, Denver, CO, USA, 2003.

Saadaoui, K. \& Ozguler, A. B. (2005). A new method for the computation of all stabilizing controllers of a given order. International Journal of Control, Vol. 78, pp. 14-28.

Silva, G. J.; Datta, A. \& Bhattacharyya, S. P. (2001). PI stabilization of first order systems with time delay. Automatica, Vol. 37, pp. 2225-2031.

Silva, G. J.; Datta, A. \& Bhattacharyya, S. P. (2002). New results on the synthesis of PID controllers. IEEE Transactions on Automatic Control, Vol. 47, 2002, pp. 241-252.

Silva, G. J.; Datta, A. \& Bhattacharyya, S. P. (2005) PID controllers for time delay systems, Birkhauser, ISBN 978-0817642662, Boston USA.

Seuret, A.; Michaut, F.; Richard, J. P. \& Divoux, T. (2006). Network control using GPS synchronization, Proc. American Control Conference, Minneapolis, Minnesota, USA. 
Wang, D. J. (2007). Further results on the synthesis of PID controllers. IEEE Transactions on Automatic Control, Vol. 52, pp. 1127-1132.

Wang, D. J. (2007). Stabilizing regions of PID controllers for $n$-th order all pole plants with dead time. IET Control Theory \& Applications, Vol. 1, pp. 1068-1074.

Zhong, Q. C. (2006). Robust control of time delay systems, Springer-Verlag, ISBN 9781846282645. 


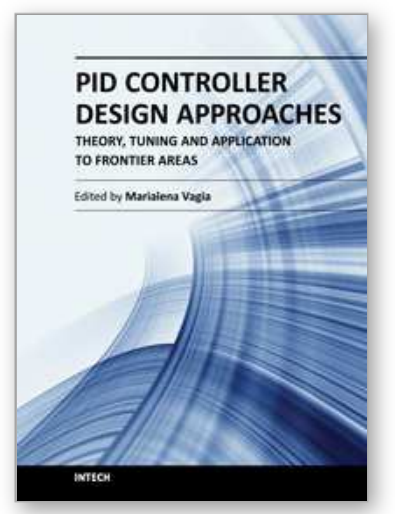

\author{
PID Controller Design Approaches - Theory, Tuning and \\ Application to Frontier Areas \\ Edited by Dr. Marialena Vagia
}

ISBN 978-953-51-0405-6

Hard cover, 286 pages

Publisher InTech

Published online 28, March, 2012

Published in print edition March, 2012

First placed on the market in 1939, the design of PID controllers remains a challenging area that requires new approaches to solving PID tuning problems while capturing the effects of noise and process variations. The augmented complexity of modern applications concerning areas like automotive applications, microsystems technology, pneumatic mechanisms, dc motors, industry processes, require controllers that incorporate into their design important characteristics of the systems. These characteristics include but are not limited to: model uncertainties, system's nonlinearities, time delays, disturbance rejection requirements and performance criteria. The scope of this book is to propose different PID controllers designs for numerous modern technology applications in order to cover the needs of an audience including researchers, scholars and professionals who are interested in advances in PID controllers and related topics.

\title{
How to reference
}

In order to correctly reference this scholarly work, feel free to copy and paste the following:

Karim Saadaoui, Sami Elmadssia and Mohamed Benrejeb (2012). Stabilizing PID Controllers for a Class of Time Delay Systems, PID Controller Design Approaches - Theory, Tuning and Application to Frontier Areas, Dr. Marialena Vagia (Ed.), ISBN: 978-953-51-0405-6, InTech, Available from:

http://www.intechopen.com/books/pid-controller-design-approaches-theory-tuning-and-application-to-frontierareas/stabilizing-pid-controllers-for-a-class-of-time-delay-systems

\section{INTECH}

open science | open minds

\section{InTech Europe}

University Campus STeP Ri

Slavka Krautzeka 83/A

51000 Rijeka, Croatia

Phone: +385 (51) 770447

Fax: +385 (51) 686166

www.intechopen.com

\section{InTech China}

Unit 405, Office Block, Hotel Equatorial Shanghai

No.65, Yan An Road (West), Shanghai, 200040, China

中国上海市延安西路65号上海国际贵都大饭店办公楼 405 单元

Phone: +86-21-62489820

Fax: $+86-21-62489821$ 
(C) 2012 The Author(s). Licensee IntechOpen. This is an open access article distributed under the terms of the Creative Commons Attribution 3.0 License, which permits unrestricted use, distribution, and reproduction in any medium, provided the original work is properly cited. 